\title{
Autonomous distributed temperature sensing for long-term heated applications in remote areas
}

\author{
A.-M. Kurth ${ }^{1,2}$, N. Dawes ${ }^{3}$, J. Selker ${ }^{4}$, and M. Schirmer ${ }^{1,2}$ \\ ${ }^{1}$ Department of Water Resources and Drinking Water, Eawag, Swiss Federal Institute of Aquatic Science and Technology, \\ Überlandstrasse 133, 8600 Dübendorf, Switzerland \\ ${ }^{2}$ The Center of Hydrogeology and Geothermics (CHYN), University of Neuchâtel, Rue Emile-Argand 11, \\ 2000 Neuchâtel, Switzerland \\ ${ }^{3}$ Department of Snow and Permafrost, WSL Institute for Snow and Avalanche Research SLF, Flüelastrasse 11, \\ 7260 Davos Dorf, Switzerland \\ ${ }^{4}$ Department of Biological and Ecological Engineering, Oregon State University, 210 Gilmore Hall, \\ Corvallis, Oregon 97331, USA
}

Correspondence to: A.-M. Kurth (anne-marie.kurth@eawag.ch), N. Dawes (dawes@slf.ch), J. Selker (selkerj@engr.orst.edu), and M. Schirmer (mario.schirmer@eawag.ch)

Received: 7 September 2012 - Published in Geosci. Instrum. Method. Data Syst. Discuss.: 22 October 2012 Revised: 18 January 2013 - Accepted: 26 January 2013 - Published: 7 February 2013

\begin{abstract}
Distributed temperature sensing (DTS) is a fiberoptical method enabling simultaneous temperature measurements over long distances. Electrical resistance heating of the metallic components of the fiber-optic cable provides information on the thermal characteristics of the cable's environment, providing valuable insight into processes occurring in the surrounding medium, such as groundwater-surface water interactions, dam stability or soil moisture. Until now, heated applications required direct handling of the DTS instrument by a researcher, rendering long-term investigations in remote areas impractical due to the often difficult and time-consuming access to the field site. Remote control and automation of the DTS instrument and heating processes, however, resolve the issue with difficult access. The data can also be remotely accessed and stored on a central database. The power supply can be grid independent, although significant infrastructure investment is required here due to high power consumption during heated applications. Solar energy must be sufficient even in worst case scenarios, e.g. during long periods of intense cloud cover, to prevent system failure due to energy shortage. In combination with storage batteries and a low heating frequency, e.g. once per day or once per week (depending on the season and the solar radiation on site), issues of high power consumption may be resolved. Safety regulations dictate adequate shielding
\end{abstract}

and ground-fault protection, to safeguard animals and humans from electricity and laser sources. In this paper the autonomous DTS system is presented to allow research with heated applications of DTS in remote areas for long-term investigations of temperature distributions in the environment.

\section{Introduction}

Temperature measurements have been recognized to be a powerful tool in natural sciences (Leap, 2006; Schneider, 1962). They may be employed to investigate hydrologic systems (Selker et al., 2006a), stream dynamics (Selker et al., 2006b), groundwater-surface water interactions (Slater et al., 2010), atmospheric processes (Keller et al., 2011; Thomas et al., 2012) or soil moisture (Sayde et al., 2010; SteeleDunne et al., 2010; Striegl and Loheide II, 2012). Fiber-optic temperature measurements may also be used in engineering, e.g. for the detection of illicit sewage connections (Hoes et al., 2009), hot spot identification in power cables (Yilmaz and Karlik, 2006) or the investigation of dam stability (Wang et al., 2010).

There are several ways to investigate temperature distribution patterns, the most common being locally logged temperature probes (Anderson, 2005; Schmidt et al., 2006; Gardner 
et al., 2011). While these devices provide accurate point measurements of temperature, they have several disadvantages when utilized in the monitoring of extended areas, i.e. when more than 10 data loggers are in use: (i) data collection is very time consuming, as each data logger has to be manually retrieved; (ii) each data logger provides one individual data set, leading to a multitude of records; (iii) data handling is relatively time consuming as data sets have to be combined prior to data analysis; and (iv) sensor calibration is only possible at the start and end of installations, thus mid-installation data are subject to possible sensor drift. Additionally, the acquisition of large numbers of data loggers is quite costly.

Alternatively, distributed temperature sensing (DTS) measures temperature along a fiber-optic cable, thereby allowing (i) the simultaneous collection of distributed temperature data, (ii) over long distances, with (iii) a temperature resolution of $\pm 0.01^{\circ} \mathrm{C}$ under ideal conditions (e.g. Selker et al., 2006a).

DTS is a fiber-optical method for temperature determination along a fiber-optic cable connected to a DTS instrument. The fiber-optic cables utilized in environmental applications usually consist of one or several protected glass fibers. The required protection depends greatly on the environment of installation; however, when crushing risks are present this often includes metal armoring, and almost always an outer protective plastic sheath for protection against water, corrosion, and for electrical isolation. The DTS instrument's main components are (i) a laser and (ii) a detector. During a temperature measurement a laser pulse is sent into the fiber-optic cable; the photons of the laser pulse are backscattered within the glass fiber and analyzed (i) for their energy and (ii) time of arrival. The energy of the photons indicates their backscatter history: no loss of energy indicates elastic backscattering of the photons, whereas loss or gain in energy indicates inelastic backscattering (Smith et al., 2007). In Raman-based DTS temperature measurements, the ratio of the number of inelastically backscattered photons with energy above and below the injected light wavelength is applied to calculate the temperature of the fiber-optic cable, as the backscatter intensity largely depends on the vibrational level of the interacting molecule (Smith et al., 2007) and therefore on the temperature of the silica glass in the glass fiber of the fiberoptic cable. The DTS therefore measures the temperature of the surroundings by determining the temperature of the glass fiber. Here it is assumed that the fiber-optic cable is in thermal equilibrium with the surrounding medium. The resolution of the time of arrival of the photons by the detector allows the allocation of the measured temperature to a specific section of the fiber-optic cable. Temperature measurements with the DTS are spatial averages rather than point measurements, as the temperature reported at point $x$ is actually the integration of all photons being backscattered in the section of cable about the point $x$ within a distance defined as the instrument resolution. Currently available commercial Ramanbased DTS systems have resolutions of between 0.3 and
$4.0 \mathrm{~m}$. Nonetheless, the temperature distribution along the whole length of the fiber-optic cable can be determined in a single measurement, thereby replacing a large number of data loggers. This shows the practicality of DTS for temperature monitoring over long distances and thus large areas.

DTS is most often employed in passive mode, i.e. the unaltered ambient temperature is determined along the fiberoptic cable. The DTS may also be used in an active mode, in which the response to injection of heat is studied, for instance following thermal resistance heating of the metal armor surrounding the fiber-optic cable. This provides additional information, not only on (i) the background temperature of the fiber-optic cable prior to its heating, but on (ii) its heating and cooling behavior, which again reflects the conditions in the environment of the fiber-optic cable. So far, this technique has been used to investigate dam stability (Etzer et al., 2012), soil moisture (Sayde et al., 2010), and hyporheic zone processes (articles in preparation).

The heating of the fiber-optic cable usually has to be directly handled by an operator, rendering it impractical for long-term measurements, particularly in remote areas. In remote settings, e.g. mountainous regions, which might be difficult to access and without power infrastructure, long-term active, heated DTS applications have previously been difficult to implement due to the time consuming data collection. However, as seen in the previous paragraph, there are numerous applications for active DTS in these areas, particularly in the environmental sciences and engineering. An autonomous DTS system, i.e. a DTS system for heated applications that is independent of the physical presence of an operator, is therefore required to enable research in scientifically interesting remote areas. One future application of the autonomous DTS system will be the investigation of the effect of river restoration on hyporheic exchange in Swiss rivers and streams.

The autonomous DTS system proposed here consists of various components that enable remote access to the DTS, collection of the temperature data and control of the heating of the fiber-optic cable. These components are (i) a computer, (ii) a control box for the automated heating of the fiber-optic cable, and (iii) a remote connection, such as a $3 \mathrm{G}$ modem or wireless connection. Another, optional, feature is a gridindependent power supply, such as solar panels or wind generators. However, there are several issues with the development of an autonomous DTS system depending on the DTS type and measurement location: (a) the remote control of the DTS has to be compatible with the DTS instrument and the heating control box, safe and stable, and available in undeveloped or remote areas without internet or mobile phone connection; (b) the power consumption of the heating of the fiber-optic cable can be high, depending on the material and the length of the fiber-optic cable, which are difficult to provide with grid-independent power supplies; (c) the high voltage of the heating might be dangerous to humans and animals, particularly rodents; and (d) the power connections to the fiber-optic cable have to be safe, i.e. the fiber-optic 
cable has to be of a specific type to enable standard electrical connections.

This paper provides guidance on how to upgrade a standard DTS to an autonomous DTS system, enabling long-term passive and active heated DTS temperature measurements in remote areas without a direct operator.

\section{Instrumental set-up}

\subsection{System components}

The autonomous DTS system consists of various building blocks: (i) the passive DTS set-up, (ii) components for the active, heated, employment of the DTS, and (iii) the control/communication components. The separate components for each of the aforementioned three building blocks will be described in more detail in the subsections below.

\subsubsection{Passive DTS components}

The basic components required for the passive employment of the DTS instrument are (i) the DTS instrument, (ii) a computer for data storage and DTS operation, (iii) a fiber-optic cable, and (iv) temperature controlled enclosures for reference temperature measurements ("water baths"), required for the calibration of the DTS instrument.

The DTS instrument and the fiber-optic cable are the two most important components of any autonomous DTS system. Prior to purchase of a DTS instrument there are a number of things to be considered: (i) the type and (ii) location of the experiment, (iii) the data resolution, particularly with regard to spatial, temporal and temperature resolution, and (iv) the data format. The type and location of the experiment define the demand on the DTS instrument, such as long-term or short-term, indoor or outdoor experiments, high or low temperatures, dry or humid climates, corrosive environments, occurrence of rock slides or avalanches, and other conditions that might damage the instrument. Several manufacturers offer instruments for temperature ranges of -40 to $+65^{\circ} \mathrm{C}$ and provide steel enclosures for the instruments, to allow for installation without further environmental protection. The DTS instruments differ in many important regards, amongst others, in (i) the intensity and pulse-length of the laser, (ii) the sensitivity, signal-to-noise, and temporal resolution of the detector system, (iii) the wavelength of the laser, and (iv) the data storage capabilities and interpretation software. Hence, the temperature and spatial resolution, as well as the signalto-noise ratio, and therefore the data quality, of the various DTS instruments differ considerably (e.g. each by over a factor of 10) and have to be carefully evaluated prior to purchase of the DTS instrument.

For DTS systems without built-in computer control and data storage, there are a number of suitable computers to choose from, ranging from small PCs to notebooks, the choice depending on the requirements of the experimental set-up. However, the main point to consider is power consumption and performance reliability, as well as ruggedness, particularly with regard to climatic conditions. As the data acquisition is hardware-based, the computational capabilities of the PC are never limiting. A key consideration is how the computer responds to a power outage. In remote applications the ability to program the computer to autonomously reboot to a fully functioning data collection state is essential. In order to connect to the network, either a mobile network modem (GPRS/3G/UMTS/LTE) or a Wi-Fi card is required according to the scenario. We have found many integrated options for this, but a USB solution is also possible.

\subsubsection{Cable considerations}

As mentioned above, the fiber-optic cable is one of the most important components of any autonomous DTS system. It has to be responsive enough to rapidly resolve minute changes in temperature yet strong enough to withstand tensile stress and strain, crushing and bending, as well as solar radiation and environmental stresses, such as corrosive fluids or extreme temperatures. Additionally, for active deployments the fiber-optic cable must have metal components to enable thermal resistance heating via application of electrical currents. The voltage may be applied either across the steel armor or across supplementary copper wires (Freifeld et al., 2008; Sayde et al., 2010). Heating of the fiber-optic cable via the steel armor is suitable for short fiber-optic cable sections up to $250 \mathrm{~m}$, whereas heating via copper wires is suitable for longer fiber-optic cable sections. The copper wires may be either (i) directly integrated in the steel armor or (ii) wound separately around the central glass fiber core. The first type of fiber-optic cable is highly stress and crush resistant, but more difficult to electronically connect to the power source, while the second type of fiber-optic cable can be easily and safely connected like any other electrical cable, but may be prone to microbending issues due to the copper wires pressing on the central glass fiber core, particularly when buried in coarse sediment (unpublished data).

In general, the temperature change in the fiber-optic cable largely depends on the material and the length of its metal armor. It may be estimated by calculating the electrical resistance $R$ (Eq. 1) and, with Joule's heating law, the power $P$ (Eq. 2) applied to the metal armor of the fiber-optic cable in dependence of the power source:

$R=\rho(l / A)$

Thereby, $R$ is the electrical resistance in Ohm $[\Omega], \rho$ the electrical resistivity in $[\Omega \mathrm{m}], l$ the length of the conducting medium in $[\mathrm{m}]$, and $A$ the cross sectional area of the conducting medium in $\left[\mathrm{m}^{2}\right]$ (Griffiths, 2008). The electrical resistivity $\rho$ of austenitic stainless steel is $8.11 \times 10^{-7} \Omega \mathrm{m}$ at $26.85^{\circ} \mathrm{C}$ (Hust and Giarratano, 1975); copper has a $\rho$ of $1.68 \times 10^{-8} \Omega \mathrm{m}$ at $20^{\circ} \mathrm{C}$ (Griffiths, 2008). The power delivered by a heating system is given by 
$P=V^{2} / R$

where $P$ is the power in Watts [W] and $V$ is the voltage in Volt [V].

Given these formulas it is possible to calculate the power applied to the conducting medium as a function of the cable design and operating conditions and therefore get an estimate of the thermal energy delivery to the armor of the fiber-optic cable.

The application of these relationships can be seen by considering several typical scenarios: (i) a cable length of $50 \mathrm{~m}$, with a diameter of $1 \mathrm{~mm}^{2}$ of copper strands, a $\rho$ of $1.68 \times 10^{-8} \Omega \mathrm{m}$ and a voltage of $230 \mathrm{~V}$; (ii) the same scenario, but with a voltage of $24 \mathrm{~V}$; (iii) and (iv) the same scenarios, but with a cable length of $500 \mathrm{~m}$; (v) to (viii) as in scenarios (i) to (iv), but with an austenitic stainless steel armor instead of copper wires. The results are shown in Table 1.

In summary we see that heat delivery, per meter, increases with the square of applied voltage, and decreases with the square of cable length (since total power delivered increases linearly, as does the number of meters over which that energy is divided). Thus short fiber-optic cables with a copper armor can be heated at low voltage, and long cables with a stainless steel armor require high voltage. Tests at the River Thur in Switzerland have shown that a $1500 \mathrm{~m}$ copper strand with a total cross-section of $0.85 \mathrm{~mm}^{2}$ wrapped around a $250 \mathrm{~m}$ long fiber-optic cable reached a maximum change in temperature of around 2 Kelvin $(\mathrm{K})$ in flowing water with a temperature of 15.5 to $18.0^{\circ} \mathrm{C}$ when heated for $15 \mathrm{~min}$ at $230 \mathrm{~V}$. However, this degree of heating would differ according to the diameter of the fiber-optic cable and the thermal heat capacity of the cable and surrounding material, and if flowing water was present. Hence, pre-tests regarding the heating behavior must be performed prior to the field installation to evaluate the heating behavior in the surrounding medium, e.g. water, soil, gravel, etc. In general, the fiber-optic cable should not be kept in loops or on cable drums during heated applications, as this might cause overheating of the metal armor of the cable, leading to cable damage and potentially cable fires. In order to evade this risk only small sections of the fiber-optic cable may be heated. This would exclude sections particularly vulnerable to heating, such as cable sections kept on a cable drum or in tight loops. For safety reasons, an autonomous DTS system should have an alarm that automatically switches off the power, and thereby stops heating of the fiber-optic cable, when temperatures reach a critical level, e.g. the $85^{\circ} \mathrm{C}$ which outdoor cables typically resist whilst in continuous operation.

\subsubsection{DTS calibration reference baths}

The DTS instrument has to be carefully calibrated in order to acquire reliable temperature data (e.g. Hausner et al., 2011; van de Giesen et al., 2012). The instrument is calibrated prior to the measurement, but always requires post-measurement
Table 1. Examples of heating parameters ${ }^{\mathrm{a}}$.

\begin{tabular}{|c|c|c|c|c|c|}
\hline Scenario & $\begin{array}{c}\text { Cable } \\
\text { length } \\
(\mathrm{m})\end{array}$ & $\begin{array}{l}\text { Voltage } \\
\text { (V) }\end{array}$ & $\begin{array}{c}\text { Electrical } \\
\text { resistance }^{\mathrm{b}} \\
(\Omega)\end{array}$ & $\begin{array}{l}\text { Power } \\
\text { (W) }\end{array}$ & $\begin{array}{l}\text { Power/m } \\
\left(\mathrm{W} \mathrm{m}^{-1}\right)\end{array}$ \\
\hline \multicolumn{6}{|c|}{ Armor material copper ${ }^{\mathrm{d}}$} \\
\hline (i) & 50 & 230 & 0.8 & 62976 & $(1259.5)$ \\
\hline (ii) & 50 & 24 & 0.8 & 686 & 13.7 \\
\hline (iii) & 500 & 230 & 8.4 & 6298 & 12.6 \\
\hline (iv) & 500 & 24 & 8.4 & 69 & $(0.1)$ \\
\hline \multicolumn{6}{|c|}{ Armor material stainless steel ${ }^{\mathrm{e}}$} \\
\hline (v) & 50 & 230 & 40.6 & 1305 & 26.1 \\
\hline (vi) & 50 & 24 & 40.6 & 14 & $(0.28)$ \\
\hline (vii) & 500 & 230 & 405.5 & 130 & $(0.26)$ \\
\hline (viii) & 500 & 24 & 405.5 & 1 & $(0.003)$ \\
\hline
\end{tabular}

${ }^{a}$ Heating parameters describing heating efficiency of the metal armor in fiber-optic cables: electrical resistance $R$, and power $P$.

${ }^{\mathrm{b}}$ Cross sectional area $A$ of the conducting material is $10^{-6} \mathrm{~m}^{2}$.

${ }^{c}$ Power $/ \mathrm{m}$ should be $100 \mathrm{~W} \mathrm{~m}^{-1}>$ power $/ \mathrm{m}>0.5 \mathrm{~W} \mathrm{~m}^{-1}$; values above and below these thresholds are too high or low for thermal resistance heating, respectively.

${ }^{\mathrm{d}}$ Electrical resistivity of copper $\rho_{\mathrm{Cu}} 1.68 \times 10^{-8} \Omega \mathrm{m}$ at $20^{\circ} \mathrm{C}$ (Griffiths, 2008).

e Electrical resistivity of $20 \%$ nickel $/ 16 \%$ chromium austenitic stainless steel $\rho_{\text {SS }}$ $8.11 \times 10^{-7} \Omega \mathrm{m}$ at $26.85^{\circ} \mathrm{C}$ (Hust and Giarratano, 1975).

re-calibration to obtain the highest precision data. In both cases well-mixed insulated water baths are required as reference sections in which the temperature of the fiber-optic cable can be assumed to be constant over the period of calibration data collection (typically $<15 \mathrm{~min}$ ). One calibration bath can be constructed from wetted ice, though care must be taken that the ice fully envelopes the test section, as otherwise the average temperature cannot be guaranteed to be $0{ }^{\circ} \mathrm{C}$. As it is impossible to install and maintain an ice bath in a remote setting, though, two continuously mixed water baths at different temperatures may be used instead, e.g. one water bath may be filled with river water, the other water bath may be filled with river water and a small heating device such as used in fish ponds to prevent freezing over in winter. Reference baths must be equipped with additional temperature probes, so that the actual water temperature is known and can be used to calibrate the temperature of the fiber-optic cable section in the respective water baths. Some DTS systems are provided with external temperature probes; however, these are not considered to be as precise or reliable as required for such applications. It is advisable to use two water baths at each end of the fiber-optic cable, warmer and colder than the monitored temperatures, so as to avoid error in temperature measurements due to a decrease in signal quality as caused by increasing loss of light over the whole length of the fiber-optic cable. General guidance on instrument calibration is given by Hausner et al. (2011) for single-ended installations and van de Giesen et al. (2012) for double-ended installations. 


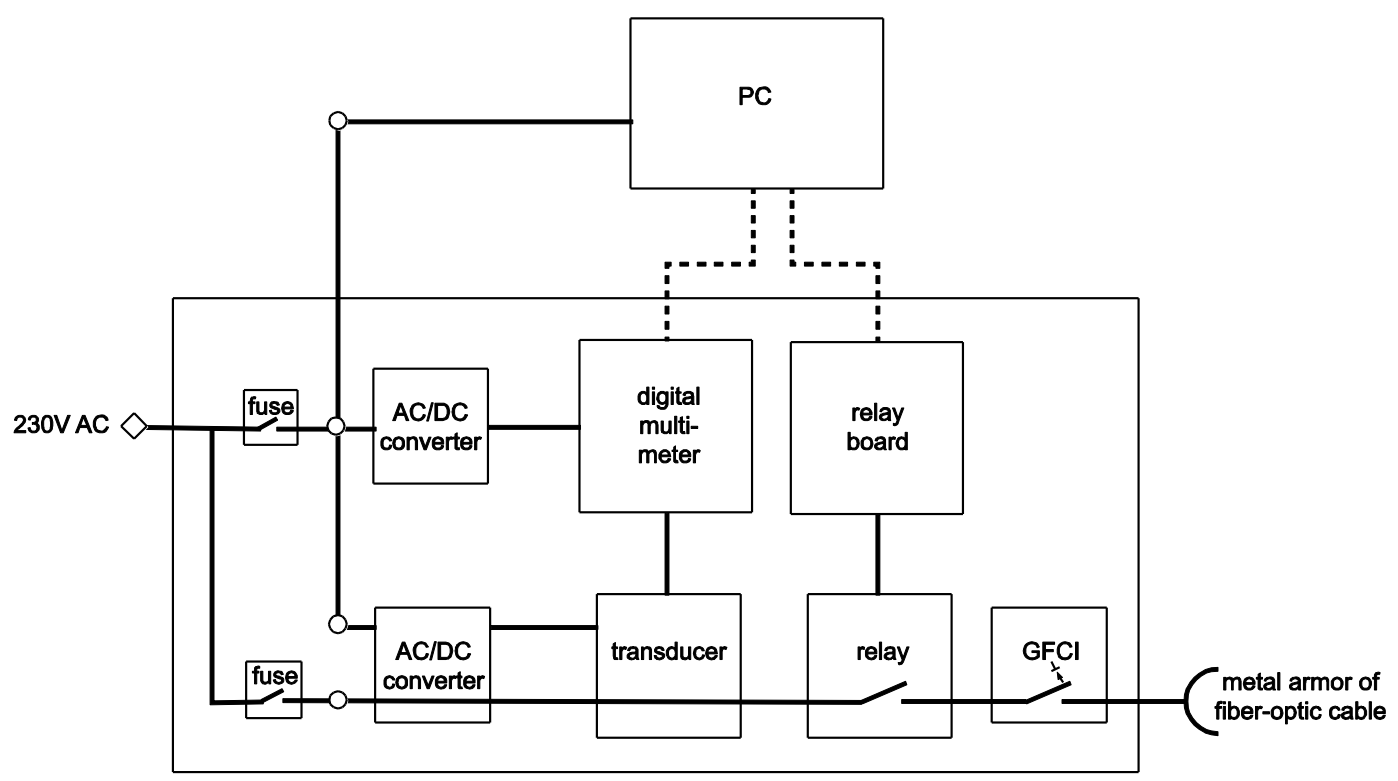

Fig. 1. Schematic diagram of the heating control for automated heating of a fiber-optic cable in the autonomous DTS system. Bold lines represent power connections, dashed lines are data connections with the PC; the box marked GFCI represents the ground-fault circuit interrupter.

\subsubsection{Active DTS components}

The two components required for an active employment of the DTS instrument in an autonomous system are (i) a control box for the automated heating of the fiber-optic cable, and (ii) a (grid-independent) power supply.

The main features of the control box are (i) a time switch (here in form of a relay board and a relay) that automatically controls the electrical current, and thus the heating of the fiber-optic cable, and (ii) a digital multimeter to record the actual current flowing through the metal armor of the fiber-optic cable, so as to be able to correlate heating behavior and voltage applied. Both time switch and digital multimeter are controlled via the PC. Another important feature of the control box is a ground-fault circuit interrupter, also known as residual-current device, which switches off the current in case there is a difference in current between the energized conductor and the return neutral conductor, e.g. caused by a person accidentally touching the energized part of the conductor. In the control box the ground-fault circuit interrupter has to be placed between the relay and the metal armor of the fiber-optic cable (Fig. 1). A potential issue with the ground-fault circuit interrupter is the leakage of electricity through the fiber-optic cable's insulation, which might cause the ground-fault circuit interrupter to trip. Although an increase in the tripping current would prevent tripping of the ground-fault circuit interrupter, it would also increase the risk of harmful electrical shocks. Therefore, individual solutions have to be found to trade between security and scientific aim. Additionally, fuses have to be installed to protect the equipment from overcurrent or short-circuit conditions. The other features in the control box are AC/DC converters altering the provided voltage into the voltage required by the consumer loads; a transducer converting the current into a voltage to enable the digital multimeter to read the actual current flown through the metal armor of the fiber-optic cable; a relay board enabling the switching function of the PC; and a relay to actually switch the current on and off. Please note that the system is shown for a $230 \mathrm{~V}$ power supply. In the case where a grid-independent power supply is used with a lower voltage, power converters have to be installed.

Powering the autonomous DTS system in remote areas may well be an issue, as power lines might not run in close vicinity or might not be accessible. Hence, the autonomous DTS system should be powered by grid-independent power supplies, such as solar panels or wind and water turbines. As mentioned in the previous section, ground-fault circuit interrupters have to be placed in between the power source, e.g. the battery of the solar panels, and the consumer loads, e.g. the control box, the DTS or the PC, to prevent accidental electrocution.

The energy demand for the heated cable method presents a critical challenge for remote off-grid powered operations. Typical heating rates for DTS methods were between 3 and $20.5 \mathrm{~W} \mathrm{~m}^{-1}$ (Striegl and Loheide II, 2012; Sayde et al., 2010; Freifeld et al., 2008). Earlier tests with $1500 \mathrm{~m}$ copper strands wrapped around a $250 \mathrm{~m}$ long fiber-optic cable at the River Thur in Switzerland indicated a power consumption of $869 \mathrm{~W}$ at $230 \mathrm{~V}\left(0.58 \mathrm{~W} \mathrm{~m}^{-1}\right)$. This was sufficient to generate a maximum temperature change of $2 \mathrm{~K}$ in the metal armor of the fiber-optic cable section surrounded by water following a 15 minute heat pulse (unpublished data). This power demand 
is difficult to supply in a remote field site without access to the power grid. Hence, the fiber-optic cable has to be heated at a higher voltage, but for shorter sequences. Currently, the most feasible options include solar panels or the combination of solar panels and wind generators.

The power supply is highly dependent on the power consumption of (i) the DTS instrument, (ii) the additional components, such as the heating control box, the PC and the time switch, and (iii) the type and length of the fiber-optic cable. The energy demand may be calculated with Eqs. (1) and (2) provided in Sect. 2.1.2. Generally, at lower voltage, fiber-optic cables will have to be heated for longer periods to achieve a measureable change in temperature and might have to be divided into subsections, e.g. four $200 \mathrm{~m}$-subsections, rather than one $800 \mathrm{~m}$ long section. However, as mentioned above, this is highly dependent on the type and length of the fiber-optic cable.

\subsubsection{Autonomous DTS system components}

The building blocks that finally turn the components described so far into an autonomous DTS system are (i) a device for remote access to the DTS and the control box via the control PC, and (ii) a script/software to enable automated data transfer to a central server, from which the data can be accessed from anywhere in the world.

The DTS will typically be controlled using a low powered Windows embedded PC with a cellular modem (e.g. 3G). To allow DTS control, the system will typically be accessed over a Virtual Network Computing (VNC) connection from a remote location, such that the native software for the DTS can be used. Data may be retrieved via File Transfer Protocol (FTP). In order to make the data available to a wide audience of collaborators, it might be uploaded directly onto a publicly accessible platform. The authors chose to store and access the data using Global Sensor Network (GSN) middleware (Aberer et al., 2006), which includes web-services and a web-based query download interface, and interfaces to the Open Support Platform for Environmental Research (OSPER). For areas where mobile network coverage is unavailable, a stand-alone Wi-Fi access point may be placed in an area with network coverage and the windows embedded PC hence connected to the Internet over a directional Wi-Fi link. In many cases, this access point will also need to be grid independent and therefore low power. Several options are available, but two options used by the authors are a MikroTik router box; or a PC engines mini-PC with MikroTik RouterOS. The use of RouterOS was chosen to significantly ease programming of the router. A guide can be found at http://www.swiss-experiment.ch/index.php/ SwissEx:Wifi. A directional Wi-Fi antenna should be used to enable communications. Hereby, greater directionality provides higher signal gain, and thus enables communications over long distances. Good results have been gained by the authors using both 2.4 and $5.7 \mathrm{GHz}$; however, $2.4 \mathrm{GHz}$ Wi-Fi allows more flexibility in the hardware used. Wi-Fi cards for the computer/router at each end and the corresponding antenna type should be chosen accordingly. This set-up provides direct access to the PC controlling the DTS instrument and the heating control, thus enabling their remote control, as well as access to the data collected during passive and active measurement periods.

\subsection{System assembly}

The building blocks of the autonomous DTS System will be assembled as follows: all components, i.e. the DTS instrument, the PC, the modem and the control box are connected to the power supply; the PC is connected to the DTS instrument, the modem and the control box and the DTS instrument and the control box are connected to the fiber-optic cable. It is advisable to store the autonomous DTS System in a lockable metal box, to avoid causing harm to humans or animals and reduce the risk of environmental impacts, such as hail, frost or humidity, damaging the system.

\section{Summary}

The upgrade of a passive DTS instrument to an active, autonomous DTS system is presented. Autonomous DTS systems enable passive and active, heated long-term temperature measurements over distances of a few hundred meters in remote areas without human presence, which will enable measurements in interesting, but difficult to reach far-off areas. The remarkable features of the autonomous DTS system are (a) that it is remote controlled, (b) has an automated data transfer, and (c) can be built to be grid independent. Hence, the system is truly independent of a direct operator at the field site and can be stationed in areas that are difficult to access.

There are several components required to upgrade the standard passive DTS to an autonomous DTS system. Additional to the passive DTS instrument, consisting of (i) a DTS instrument, (ii) a PC, (iii) one or more fiber-optic cables, and (iv) several water baths as temperature references, the autonomous DTS system is equipped with (v) a control box for the automated heating of the fiber-optic cable, (vi) a remote connector, such as a $3 \mathrm{G}$ modem, and software for automated data transfer, and (vii) a grid-independent power supply. The grid-independent power supply is the issue most difficult to solve, as power consumption of the heated DTS application is rather high and challenging when exclusively provided with grid-independent power supplies, such as solar panels. 
Acknowledgements. This work was funded by the Competence Center Environment and Sustainability (CCES) of the ETH domain in the framework of the Restored Corridor Dynamics (RECORD) project, the follow up project RECORD Catchment, and Swiss Experiment. The authors are grateful to the technical support of Erich Eschmann, Peter Gäumann, Silvio Harndt, and Andy Raffainer.

Edited by: A. Benedetto

\section{References}

Aberer, K., Hauswirth, M., and Salehi, A.: A middleware for fast and flexible sensor network deployment, paper presented at the Very Large Data Bases (VLDB) Conference, Seoul, Korea, 2006.

Anderson, M. P.: Heat as a Ground Water Tracer, Groundwater, 43, 951-968, doi:10.1111/j.1745-6584.2005.00052.x, 2005.

Etzer, T., Aufleger, M., and Dornstädter, J.: The Use of Distributed Fiber Optic Measurements in Hydraulic Engineering, paper presented at HydroVision Russia Conference and Exhibition, Moscow, Russia, 2012.

Freifeld, B. M., Finsterle, S., Onstott, T. C., Toole, P., and Pratt, L. M.: Ground surface temperature reconstructions: Using in situ estimates for thermal conductivity acquired with a fiber-optic distributed thermal perturbation sensor, Geophys. Res. Lett., 35, L14309, doi:10.1029/2008GL034762, 2008.

Gardner, W. P., Susong, D. D., Solomon, D. K., and Heasler, H. P.: A multitracer approach for characterizing interactions between shallow groundwater and the hydrothermal system in the Norris Geyser Basin area, Yellowstone National Park, Geochem. Geophy. Geosy., 12, Q08005, doi:10.1029/2010GC003353, 2011.

Griffiths, D. J.: Electrodynamics, in Introduction to Electrodynamics, Pearson Benjamin Cummings, San Francisco, CA, 285-342, 2008.

Hausner, M. B., Suárez, F., Glander, K. E., van de Giesen, N., Selker, J. S., and Tyler, S. W.: Calibrating Single-Ended FiberOptic Raman Spectra Distributed Temperature Sensing Data, Sensors, 11, 10859-10879, doi:10.3390/s111110859, 2011.

Hoes, O. A. C., Schilperoort, R. P. S., Luxemburg, W. M. J., Clemens, F. H. L. R., and van de Giesen, N. C.: Locating illicit connections in storm water sewers using fiber-optic distributed temperature sensing, Water Res., 43, 5187-5197, doi:10.1016/j.watres.2009.08.020, 2009.

Hust, J. G. and Giarratano, P. J.: Thermal Conductivity and Electrical Resistivity Standard Reference Materials: Austenitic Stainless Steel, SRM's 735 and 798, from 4 to 1200 K, US National Bureau of Standards Special Publication 260-46, US National Bureau of Standards, Washington, DC, 1975.

Keller, C. A., Huwald, H., Vollmer, M. K., Wenger, A., Hill, M., Parlange, M. B., and Reimann, S.: Fiber optic distributed temperature sensing for the determination of the nocturnal atmospheric boundary layer height, Atmos. Meas. Tech., 4, 143-149, doi:10.5194/amt-4-143-2011, 2011.

Leap, D. I.: Groundwater and Heat Flow, in: The Handbook of Groundwater Engineering, edited by: Delleur, J. W., CRC Press, Boca Raton, FL, 13-1-13-16, 2006.
Sayde, C., Gregory, C., Gil-Rodriguez, M., Tufillaro, N., Tyler, S., van de Giesen, N., English, M., Cuenca, R., and Selker, J. S.: Feasibility of soil moisture monitoring with heated fiber optics, Water Resour. Res., 46, W06201, doi:10.1029/2009WR007846, 2010.

Schmidt, C., Bayer-Raich, M., and Schirmer, M.: Characterization of spatial heterogeneity of groundwater-stream water interactions using multiple depth streambed temperature measurements at the reach scale, Hydrol. Earth Syst. Sci., 10, 849-859, doi:10.5194/hess-10-849-2006, 2006.

Schneider, R.: An Application of Thermometry to the Study of Ground Water, US Geological Survey Water-Supply Paper 1544B, US Geological Survey, Washington, 1-16, 1962.

Selker, J. S., Thévenaz, L., Huwald, H., Mallet, A., Luxemburg, W., van de Giesen, N., Stejskal, M., Zeman, J., Westhoff, M., and Parlange, M. B.: Distributed fiber-optic temperature sensing for hydrologic systems, Water Resour. Res., 42, W12202, doi:10.1029/2006WR005326, 2006a.

Selker, J., van de Giesen, N., Westhoff, M., Luxemburg, W., and Parlange, M. B.: Fiber optics opens window on stream dynamics, Geophys. Res. Lett., 33, L24401, doi:10.1029/2006GL027979, 2006b.

Slater, L. D., Ntarlagiannis, D., Day-Lewis, F. D., Mwakanyamale, K., Versteeg, R. J., Ward, A., Strickland, C., Johnson, C. D., and Lane Jr., J. W.: Use of electrical imaging and distributed temperature sensing methods to characterize surface water-groundwater exchange regulating uranium transport at the Hanford 300 Area, Washington, Water Resour. Res., 46, W10533, doi:10.1029/2010WR009110, 2010.

Smith, F. G., King, T. A., and Wilkins, D.: Interaction of Light with Matter, in Optics and Photonics: An Introduction, John Wiley \& Sons Ltd, Chichester, England, 2007.

Steele-Dunne, S. C., Rutten, M. M., Krzeminska, D. M., Hausner, M., Tyler, S. W., Selker, J., Bogaard, T. A., and van de Giesen, N. C.: Feasibility of soil moisture estimation using passive distributed temperature sensing, Water Resour. Res., 46, W03534, doi:10.1029/2009WR008272, 2010.

Striegl, A. M. and Loheide II, S.P.: Heated Distributed Temperature Sensing for Field Scale Soil Moisture Monitoring, Ground Water, 50, 340-347, doi:10.1111/j.1745-6584.2012.00928.x, 2012.

Thomas, C. K., Kennedy, A. M., Selker, J. S., Moretti, A., Schroth, M. H., Smoot, A. R., Tufillaro, N. B., and Zeeman, M. J.: Highresolution fiber-optic temperature sensing: A new tool to study the two-dimensional structure of atmospheric surface layer flow, Bound.-Lay. Meteorol., 142, 177-192, doi:10.1007/s10546-0119672-7, 2012.

van de Giesen, N., Steele-Dunne, S. C., Jansen, J., Hoes, O., Hausner, M. B., Tyler, S., and Selker, J.: Double-Ended Calibration of Fiber-Optic Raman Spectra Distributed Temperature Sensing Data, Sensors, 12, 5471-5485, doi:10.3390/s120505471, 2012.

Wang, J., Hu, C., Xu, H., Kang, J., Zhang, Z., and Gong, H.: Application research of DTS system in dam, in: Advanced Sensor Systems and Applications IV, Proceedings of SPIE, vol. 7853, edited by: Culshaw, B., Liao, Y., Wang, A., Bao, X., Fan, X., and Zhang, L., 78532L, SPIE, Bellingham, WA, 2010.

Yilmaz, G. and Karlik, S. E.: A distributed optical fiber sensor for temperature detection in power cables, Sensors Actuators A, 125, 148-155, doi:10.1016/j.sna.2005.06.024, 2006. 